\title{
DESNUTRIÇÃO, SARCOPENIA E COVID-19 NO IDOSO. EVIDÊNCIA CIENTÍFICA DA SUPLEMENTAÇÃO DE VITAMINA D
}

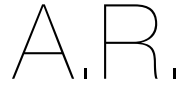
ARTIGO DE REVISÃO

1 Escola Superior de Tecnologia da Saúde de Lisboa do Instituto Politécnico de Lisboa, Av. D. João Il, Lote 4.69.01,

1990-096 Lisboa, Portuga

2 Health \& Technology Research Centre, Escola

Superior de Tecnologia da Saúde de Lisboa do Instituto Politécnico de

Lisboa,

Av. D. João II, Lote

4.69.01,

1990-096 Lisboa, Portugal

*Endereço para correspondência:

Inês Henriques

Escola Superior de Tecnologia da Saúde de Lisboa do Instituto Politécnico de Lisboa, Av. D. João II, Lote 4.69.01, 1990-096 Lisboa, Portugal 2016108@alunos.estesl.jpl.pt

Histórico do artigo:

Recebido a 27 de maio de 2020 Aceite a 29 de junho de 2020

\section{MALNUTRITION, SARCOPENIA AND COVID-19 IN THE ELDERLY. SCIENTIFIC EVIDENCE FOR VITAMIN D SUPPLEMENTATION}

Inês Henriques'; Marisa Cebola²; Lino Mendes²

\section{RESUMO}

INTRODUÇÃo: Na pandemia de COVID-19, os idosos são considerados uma população de risco acrescido. Situação que se agrava na presença de desnutrição e sarcopenia. A vitamina D pode ser uma potencial adjuvante na prevenção e tratamento de doentes com infeções virais respiratórias, que normalmente apresentam baixos níveis de vitamina $D$. A suplementação de vitamina $D$ em doentes com COVID-19, poderá ser um passo importante na prevenção e disseminação da infeção.

OBJETIVOS: Analisar a evidência sobre a suplementação de vitamina D em idosos com desnutrição, sarcopenia e COVID-19. METODOLOGIA: Analisar a literatura publicada na base de dados eletrónica Pubmed nos últimos 5 anos, utilizando as palavras-chave "covid-19", "elderly", "malnutrition", "sarcopenia" e "vitamin D". A pesquisa foi realizada entre abril e maio de 2020. RESULTADOS: A suplementação de vitamina D, em caso de carência, demonstrou um efeito benéfico na melhoria da função muscular e na redução da severidade e mortalidade por infeções respiratórias. Quando associada à proteína e ao exercício físico, apresentou um possível efeito sinérgico na quantidade e qualidade muscular. Em doentes com COVID-19, nas quais foram relatadas concentrações mais baixas de $25(\mathrm{OH}) \mathrm{D}$, verificou-se um aumento da mortalidade e da incidência da doença.

CoNCLUSõES: A evidência existente é pouco conclusiva e não é suficiente para estabelecer uma relação direta entre a deficiência de vitamina D e o risco de incorrer em COVID-19 no futuro. São necessários ensaios clínicos na população humana para estudar essa hipótese e inclusive perceber a influência da malnutrição e sarcopenia.

\section{PALAVRAS-CHAVE}

Covid-19, Desnutrição, Idosos, Sarcopenia, Vitamina D

ABSTRACT

INTRODUCTION: In the COVID-19 pandemic, older people are considered a population at increased risk. This situation is worsened in the presence of malnutrition and sarcopenia. Vitamin D can be a potential adjunct in the prevention and treatment of patients with respiratory viral infections who normally have low levels of vitamin D. Supplementation of vitamin D in patients with COVID-19, may be an important step in preventing and spreading the infection.

OBJECTIVES: Analyze the evidence on vitamin D supplementation in elderly with malnutrition, sarcopenia and COVID-19. METHODOLOGY: Analyze the literature published in the electronic database Pubmed in the last 5 years, using the keywords "covid-19", "elderly", "malnutrition", "sarcopenia" and "vitamin D". The survey was conducted between April and May 2020.

RESULTS: Vitamin D supplementation, in case of deficiency, demonstrated a beneficial effect in improving muscle function and in reducing the severity and mortality from respiratory infections. When associated with protein and physical exercise, it showed a possible synergistic effect on muscle quantity and quality. In patients with COVID-19, in which lower concentrations of $25(\mathrm{OH}) \mathrm{D}$ have been reported, there has been an increase in mortality and disease incidence.

CoNCLUSIONS: The existing evidence is not conclusive and is not sufficient to establish a direct relationship between vitamin D deficiency and the risk of incurring COVID-19 in the future. Clinical trials in the human population are needed to study this hypothesis and even to understand the influence of malnutrition and sarcopenia.

KEYWORDS

Covid-19, Elderly, Malnutrition, Sarcopenia, Vitamin D 


\section{INTRODUÇÃO}

A pandemia de COVID-19 surgiu em Wuhan, na China e desde aí tornou-se uma grande ameaça humana global (1). O coronavírus da síndrome respiratória aguda grave 2 (SARS-CoV-2) é um dos principais agentes patogénicos que atinge principalmente o sistema respiratório humano, provocando infeções severas e potencialmente fatais (2). O número de idosos infetados com COVID-19 foi aumentando no mundo, sendo considerados uma população de risco acrescido. Está provado que os doentes idosos têm uma taxa superior de infeção e de mortalidade e menos resistência, agravado pela deterioração do estado nutricional (3). A desnutrição é frequente em idosos, e resulta da ingestão ou absorção nutricionais insuficientes, que leva à alteração da composição corporal e à perda de peso não intencional (4). A perda de massa muscular (MM) e a ingestão proteica insuficiente contribui, além de outros fatores predisponentes, para o desenvolvimento de sarcopenia. A sarcopenia é caracterizada pela progressiva e generalizada redução da força muscular (FM), combinada com alterações na quantidade e qualidade musculares e performance física (5). Em associação, contribuem para perda de funcionalidade dos músculos respiratórios, imunodeficiência, permitindo a persistência vírica e o aumento de células inflamatórias nos pulmões (6).

A nutrição é o maior determinante na saúde e bem-estar da população idosa, assumindo um papel crucial na modulação da homeostase imune, no tratamento e na prevenção da desnutrição e sarcopenia, assim como na abordagem de doentes com COVID-19 (7, 8). Em idosos com desnutrição e sarcopenia, a ingestão proteica adequada, ajuda a limitar e evitar os declínios na MM, força e capacidades funcionais. Intervenções nutricionais que englobem quantidades adequadas de proteína e vitamina $\mathrm{D}$, idealmente em combinação com atividade física, são estratégias promissoras para atenuar o desenvolvimento da sarcopenia (9).

No que diz respeito à prevenção e ao tratamento, ainda não existe medicamentos ou terapias eficazes disponíveis. No entanto, sabese que numa infeção vírica, é crucial otimizar a função do sistema imunológico. Uma ingestão e absorção inadequadas conduzem à deficiência de micronutrientes, prejudicando as respostas imunológicas. Além disso, as infeções aumentam a necessidade de vários nutrientes. Existem vários micronutrientes essenciais (vitaminas A, D, C, E, B6, B12, folato, cobre, ferro, zinco e selénio) para o normal funcionamento do sistema imunológico, com potenciais relações sinérgicas entre eles. Neste contexto a correção de todas as carências nutricionais e não apenas de um nutriente é essencial para uma resposta adequada à infeção pelo SARS-CoV-2 $(10,11)$.

A literatura têm suportado o papel protetor da suplementação em vitamina $D$ na prevenção e redução do risco de infeções agudas do trato respiratório (IRA), incluindo as virais (12). A vitamina $D$ pode ser uma potencial adjuvante na proteção e tratamento de doentes com infeções virais respiratórias que normalmente apresentam baixos níveis de vitamina $D(8,13)$. Durante a pandemia de COVID-19, a toma de suplementos de vitamina $\mathrm{D}$ para aumentar as concentrações de $25(\mathrm{OH}) \mathrm{D}$ em doentes, poderá ser um passo importante na prevenção da infeção e disseminação. No entanto, a hipótese de que a suplementação de vitamina D possa reduzir o risco do SARS-CoV-2 e a incidência de morte por COVID-19 está ainda em investigação pelo que devem ser efetuados ensaios clínicos para determinar as doses apropriadas e comprovar esta hipótese (13).

Em Portugal, a carência de vitamina D [25 $(\mathrm{OH}) \mathrm{D}<20 \mathrm{ng} / \mathrm{mL}]$ é altamente prevalente $(>60 \%)$, principalmente na faixa etária do idoso (14), sendo o valor sérico médio de 42,3 nmol / L(15). Segundo o Inquérito Alimentar Nacional e de Atividade Física (IAN-AF 2015-2016) a ingestão média diária de vitamina $D$ nos idosos corresponde a $5,5 \mathrm{\mu g}$ com uma mediana de 3,7 (2,0-6,8), que quando comparado às restantes faixas etárias apresenta o valor mais baixo.(16) Comparativamente às Dietary Reference Intakes (DRI) de vitamina D para a população idosa (20 $\mu \mathrm{g} / \mathrm{dia})(17)$, a ingestão diária é bastante inferior.

À luz da atual pandemia de COVID-19, avaliou-se a evidência científica sobre o efeito da suplementação de vitamina $D$ em infeções virais e respiratórias, bem como o seu efeito na função muscular em idosos sarcopenicos e desnutridos.

\section{METODOLOGIA}

A pesquisa foi realizada na base de dados eletrónica PubMed entre abril e maio de 2020. A pesquisa abrangeu artigos publicados entre 2015 e 2020 os termos de pesquisa utilizados foram "'vitamin D" AND "elderly" AND "sarcopenia" AND "malnutrition" AND “covid-19"”. Foram incluídos artigos publicados na língua inglesa e portuguesa realizados em idosos (>65 anos), relacionados com a temática de estudo, ensaios clínicos aleatorizados e estudos de coorte. Foram excluídos os artigos de revisão e meta-análises, estudos realizados em animais, casos únicos reportados e anteriores ao ano de 2015.

\section{RESULTADOS}

Na primeira pesquisa foram recuperados 285 artigos. Após a aplicação dos vários filtros (idade, língua, anos de publicação, população humana e tipos de estudo) obtiveram-se 25 artigos. Seguidamente analisou-se o título, o resumo e o texto completo e foram selecionados 16 estudos. O objetivo desta revisão foi analisar a evidência sobre a suplementação de vitamina D em idosos com desnutrição, sarcopenia e COVID-19. Dada a inexistência de evidência que relacione os três temas principais, bem como a inexistência de ensaios em humanos que avaliem a eficácia da suplementação de vitamina $\mathrm{D}$, decidiu-se incluir os estudos que englobassem as temáticas em separado ou em contextos semelhantes. Destes 16 estudos, 6 englobaram os efeitos na função muscular (importância dos músculos respiratórios na recuperação por COVID-19), 1 em doentes malnutridos (aumenta a suscetibilidade a situações de morbilidade e mortalidade), 5 englobaram outras infeções respiratórias (importância dos músculos respiratórios na recuperação por COVID-19) e 4 incluíram a COVID-19. A duração dos estudos variou de 24 dias a 1,1 anos. Nas Tabelas 1 e 2 estão presentes os artigos mencionados.

\section{ANÁLISE CRÍTICA}

Hajj E. C, et al. (18) examinaram o efeito da suplementação de vitamina $\mathrm{D}$ na FM e MM esquelética em idosos pré-sarcopenicos em eutrofia e obesidade, com carência de vitamina D. Os resultados não mostraram associação entre a concentração sérica de 25-hidroxivitamina D (25(OH)D) e a FM, mas sim entre a concentração sérica de $25(\mathrm{OH}) \mathrm{D}, \mathrm{MM}$ apendicular e massa gorda. A obesidade e a sarcopenia estão inter-relacionadas no desenvolvimento de doenças em idosos, contribuindo para piores resultados na incapacidade física e mortalidade.

Do mesmo modo, Aoki K, et al. (19) demonstraram que a suplementação de vitamina $D$ fornece melhorias na função física, prevenindo a incapacidade física e promovendo o aumento da MM em idosos da comunidade. Para além disso, avaliaram a combinação com o exercício físico diário que promove o aumento desses efeitos. A seleção de idosos saudáveis e com maior interesse pela saúde física, poderão ter influenciado os resultados e contribuindo para uma maior adesão dos participantes. 


\begin{tabular}{|c|c|c|c|c|c|}
\hline AUTORES & ESTUDO & AMOSTRA & MÉTODOS & OBJETIVO & RESULTADOS \\
\hline $\begin{array}{l}\text { Ilie C. P, et al. (2020) } \\
\text { (30) }\end{array}$ & Comunicação & 20 países europeus & $\begin{array}{l}\text { Níveis de vit.D, número de casos } \\
\text { e mortalidade em cada país. }\end{array}$ & $\begin{array}{l}\text { Associação entre os níveis } \\
\text { de vit. D em vários países e } \\
\text { os casos e a mortalidade da } \\
\text { COVID-19. }\end{array}$ & $\begin{array}{l}\text { Os níveis de vitamina D estão } \\
\text { fortemente ligados ao número } \\
\text { de casos de COVID-19 e, } \\
\text { principalmente, à mortalidade. Os } \\
\text { idosos apresentaram maior déficit. }\end{array}$ \\
\hline $\begin{array}{l}\text { D'Avolio, A et al. } \\
\text { (2020)(31) }\end{array}$ & $\begin{array}{l}\text { Coorte retrospetivo } \\
\text { (1 março a } 14 \text { abril) }\end{array}$ & $\begin{array}{l}107 \text { doentes com } \\
\text { média de idades= } 73 \\
\text { anos }\end{array}$ & $\begin{array}{l}\text { PCR nasofaríngeo para SARS- } \\
\text { CoV-2 e concentração de } \\
25(\mathrm{OH}) \text {. }\end{array}$ & $\begin{array}{l}\text { Associação entre os níveis de } \\
25(\mathrm{OH}) \text { D e PCR-positivo para } \\
\text { COVID-19. }\end{array}$ & $\begin{array}{l}\text { Doentes com SARS-CoV-2 } \\
\text { positivo têm níveis mais baixos } \\
\text { de vit. D. }\end{array}$ \\
\hline $\begin{array}{l}\text { Asyary A, et al. } \\
(2020)(32)\end{array}$ & $\begin{array}{l}\text { Observacional ( } 2 \text { de } \\
\text { março e } 10 \text { de abril) }\end{array}$ & $\begin{array}{l}\text { Casos de COVID-19 } \\
\text { na Indonésia }\end{array}$ & $\begin{array}{l}\text { Casos diários, incidência, } \\
\text { recuperação e mortalidade de } \\
\text { COVID-19. }\end{array}$ & $\begin{array}{l}\text { Relação entre a exposição } \\
\text { solar e a incidência, morte e } \\
\text { recuperação de COVID-19. }\end{array}$ & $\begin{array}{l}\text { A exposição solar está claramente } \\
\text { associada à recuperação dos } \\
\text { doentes com COVID-19. Sem } \\
\text { relação com a ocorrência e a } \\
\text { mortalidade. }\end{array}$ \\
\hline $\begin{array}{l}\text { Hastie, C.E et al } \\
(2020)(33)\end{array}$ & Coorte & $\begin{array}{l}348598 \text { participantes } \\
\text { do UK Biobank com } \\
37-73 \text { anos }\end{array}$ & $\begin{array}{l}\text { Vitamina D (concentração de } \\
25(\mathrm{OH}) \mathrm{D})\end{array}$ & $\begin{array}{l}\text { Associação entre a } \\
\text { concentração sanguínea de vit.D } \\
\text { e o risco de COVID-19. }\end{array}$ & $\begin{array}{l}\text { A concentração de vit. D não está } \\
\text { ligada ao risco de infeção por } \\
\text { COVID-19. }\end{array}$ \\
\hline
\end{tabular}

25(OH)D: 25-hidroxivitamina D

COVID-19: Coronavírus disease 19

PCR: Polymerase Chain Reaction

SARS-CoV-2: Severe acute respiratory syndrome coronavirus 2

Tabela 2

Efeitos da Suplementação de Vitamina D

\begin{tabular}{|c|c|c|c|c|c|c|}
\hline AUTORES & ESTUDO & DURAÇÃo & AMOSTRA & INTERVENÇÃO & OBJETIVO & RESULTADOS \\
\hline $\begin{array}{l}\text { Hajj E. C, et al } \\
2018(18)\end{array}$ & ECR & 6 meses & $\begin{array}{l}128 \text { idosos com carência } \\
\text { de vit. D }\end{array}$ & $\begin{array}{l}\text { 10,000 UI de vit. D } \\
\text { (3 vezes por semana) }\end{array}$ & $\begin{array}{l}\text { Efeitos da suplementação de vitamina } \\
\text { D na FPP e MM em } \\
\text { pré-sarcopénicos. }\end{array}$ & $\begin{array}{l}\text { Efeitos significativos na MM, mas } \\
\text { não na FM. }\end{array}$ \\
\hline $\begin{array}{l}\text { Aoki A, et al. } \\
2018(19)\end{array}$ & ECR & $\begin{array}{c}24 \\
\text { semanas }\end{array}$ & 148 idosos na comunidade & $\begin{array}{l}1000 \text { Ul/dia de vit.D } \\
3 \text { séries de agachamentos } \\
\text { e exercícios de equilíbrio }\end{array}$ & $\begin{array}{l}\text { Impacto do exercício e da } \\
\text { suplementação de vit.D na função } \\
\text { física e disfunção locomotora. }\end{array}$ & $\begin{array}{l}\text { Ambos melhoraram de forma } \\
\text { independente a função física e } \\
\text { aumentaram a MM. A combinação } \\
\text { dos } 2 \text { pode potenciar os efeitos. }\end{array}$ \\
\hline $\begin{array}{l}\text { Shea K M, et } \\
\text { al } 2019(20)\end{array}$ & ECR & 12 meses & $\begin{array}{l}100 \text { idosos da comunidade } \\
\text { com carência de vit.D. }\end{array}$ & $\begin{array}{l}800-1600 \text { UI de vit. D3 } \\
\text { ou placebo }\end{array}$ & $\begin{array}{l}\text { Efeito da suplementação de vit.D no } \\
\text { poder dos membros inferiores e na } \\
\text { função. }\end{array}$ & $\begin{array}{l}\text { O aumento da concentração de } \\
\text { vitamina } D \text { não afetou os parâmetros } \\
\text { musculares. }\end{array}$ \\
\hline $\begin{array}{l}\text { Verlaan S, et } \\
\text { al 2018(21) }\end{array}$ & ECR & $\begin{array}{c}13 \\
\text { semanas }\end{array}$ & 380 idosos & $\begin{array}{l}20 \mathrm{~g} \text { WP, } 3 \mathrm{~g} \text { leucina, } \\
800 \text { UI de vit, D } \\
\text { (2 vezes/ dia) }\end{array}$ & $\begin{array}{l}\text { Efeitos da vitamina D e ingestão } \\
\text { proteica nas mudanças de MM e } \\
\text { função. }\end{array}$ & $\begin{array}{l}\text { Elevados níveis de } 25(\mathrm{OH}) \mathrm{D}(>50 \\
\mathrm{nmol} / \mathrm{L}) \text { e de ingestão proteica }(>1.0 \\
\mathrm{g} / \mathrm{kg} / \mathrm{d}) \text { contribuem para ganhos } \\
\text { superiores na MM. }\end{array}$ \\
\hline $\begin{array}{l}\text { Englund D. A, } \\
\text { et al. 2018(22) }\end{array}$ & ECR & 6 meses & $\begin{array}{l}137 \text { idosos com mobilidade } \\
\text { limitada e carência de vit.D }\end{array}$ & $\begin{array}{l}20 \mathrm{~g} \text { de Whey protein, } \\
800 \mathrm{Ul} \text { de vitamina } \mathrm{D} \\
\text { Exercício } 3 \text { vezes / semana }\end{array}$ & $\begin{array}{l}\text { Efeito da suplementação } \\
\text { hiperproteica e de vitamina D e } \\
\text { exercício na composição corporal da } \\
\text { coxa e FM. }\end{array}$ & $\begin{array}{l}\text { Melhorias na composição corporal, } \\
\text { gordura subcutânea, gordura } \\
\text { intermuscular e medidas de força. }\end{array}$ \\
\hline $\begin{array}{l}\text { Yamada M, et } \\
\text { al 2019(23) }\end{array}$ & ECR & $\begin{array}{c}12 \\
\text { semanas }\end{array}$ & 112 idosos sarcopenicos & $\begin{array}{l}10 \text { g WP, } 800 \text { Ul vit.D } \\
\text { (depois do PA) } \\
30 \text { min ex ( } 2 \text { vezes/semana) }\end{array}$ & $\begin{array}{l}\text { Efeito do exercício de resistência e da } \\
\text { suplementação proteica com vit.D no } \\
\text { músculo esquelético. }\end{array}$ & $\begin{array}{l}\text { Efeito sinergético do treino de } \\
\text { resistência e da proteína na qualidade } \\
\text { muscular e FM. }\end{array}$ \\
\hline $\begin{array}{l}\text { Merker M, et } \\
\text { al 2019(24) }\end{array}$ & Coorte & 180 dias & 828 doentes hospitalizados & $\begin{array}{l}\text { Carência }<50 \mathrm{nmol} / \mathrm{L} \\
\text { Com ou sem } \\
\text { suplementação }\end{array}$ & $\begin{array}{l}\text { Estudar a prevalência e as } \\
\text { consequências da carência de } \\
\text { vitamina } D \text { em doentes malnutridos. }\end{array}$ & $\begin{array}{l}\text { Elevada prevalência de carência } \\
\text { de vit.D, associado ao aumento da } \\
\text { mortalidade a longo-prazo. }\end{array}$ \\
\hline $\begin{array}{l}\text { Mendes N. N, } \\
\text { et al 2019(25) }\end{array}$ & ECR & 3 meses & 47 idosos & $\begin{array}{l}100000 \text { Ul de } 25(\mathrm{OH}) \mathrm{D} \\
\text { durante } 15 \text { dias } \\
\text { Placebo }\end{array}$ & $\begin{array}{l}\text { Efeito da suplementação de vit. D } \\
\text { na melhoria da proteção contra o } \\
\text { influenza e resposta imune. }\end{array}$ & $\begin{array}{l}\text { Aumento dos níveis de TGF } \beta \text { no } \\
\text { plasma em resposta à vacinação, } \\
\text { sem aumento da produção de } \\
\text { anticorpos. }\end{array}$ \\
\hline $\begin{array}{l}\text { Ginde A. A, et } \\
\text { al 2016(26) }\end{array}$ & ECR & 1 ano & $\begin{array}{l}107 \text { idosos em cuidados } \\
\text { prolongados. }\end{array}$ & $\begin{array}{l}\text { Vit. D3 } 100000 \text { UI } \\
\text { Placebo: } 400-1,000 \text { IU/dia } \\
\text { ou } 12,000 \text { UI/ mês } \\
\text { de vit. D3 }\end{array}$ & $\begin{array}{l}\text { Eficácia e a segurança da } \\
\text { suplementação em doses elevadas } \\
\text { de vit. D na prevenção da IRA. }\end{array}$ & $\begin{array}{l}\text { Redução da incidência de IRA, } \\
\text { mas associado a um aumento das } \\
\text { quedas. }\end{array}$ \\
\hline $\begin{array}{l}\text { Sluyter D. J, et } \\
\text { al } 2017(27)\end{array}$ & ECR & 1,1 anos & 442 indivíduos (42-84 anos) & $\begin{array}{l}\text { Vit. D3 }(200000 \mathrm{UI}) \text { e a } \\
\text { cada mês } 100000 \mathrm{UI} \\
\text { Cápsula placebo }\end{array}$ & $\begin{array}{l}\text { Efeito a longo-prazo de elevadas } \\
\text { doses de suplementação de vit. D na } \\
\text { função pulmonar. }\end{array}$ & $\begin{array}{l}\text { Sem melhorias na função pulmonar, } \\
\text { mas em fumadores com carência de } \\
\text { vit. D ou asma/DPOC foi benéfico. }\end{array}$ \\
\hline $\begin{array}{l}\text { Martineau } \\
\text { R. A, et al } \\
2015(28)\end{array}$ & ECR & 1 ano & 137 idosos em lares & $\begin{array}{l}\text { Vit. D3 - } 2.4 \mathrm{mg} \text { a cada } \\
2 \mathrm{meses}+10 \mu \mathrm{g} / \mathrm{dia} \\
\text { Placebo } 2 \mathrm{meses}+ \\
10 \mu \mathrm{g} / \mathrm{dia}\end{array}$ & $\begin{array}{l}\text { Comparação entre elevadas vs } \\
\text { baixas doses de vit. D3 na prevenção } \\
\text { de IRA. }\end{array}$ & $\begin{array}{l}\text { A adição de uma dose elevada de } \\
\text { vit.D não influenciou o risco de IRA, } \\
\text { mas aumentou o risco e a duração } \\
\text { das IRS. }\end{array}$ \\
\hline $\begin{array}{l}\text { Shimizu Y, et } \\
\text { al 2018(29) }\end{array}$ & ECR & $\begin{array}{c}16 \\
\text { semanas }\end{array}$ & 252 indivíduos $45-74$ anos & $\begin{array}{l}25(\mathrm{OH}) \mathrm{D} 10 \mu \mathrm{g} / \mathrm{dia} \\
\text { Cápsula de placebo }\end{array}$ & $\begin{array}{l}\text { Efeito da ingestão de vit. D nas } \\
\text { infeções do trato respiratório superior. }\end{array}$ & $\begin{array}{l}\text { Redução da duração e da severidade } \\
\text { da infeção e melhoria na qualidade } \\
\text { de vida. }\end{array}$ \\
\hline
\end{tabular}

25(OH)D: 25-hidroxivitamina D

DPOC: Doença pulmonar obstrutiva crónica

ECR: Ensaio clínico aleatorizado

FM: Força muscular

FPP: Força de preensão palmar
IRA: Infeção respiratória aguda

IRS: Infeção do trato respiratório superior

MM: Massa magra

TGFB: Transforming growth factor beta 
Por outro lado, um estudo recente em idosos da comunidade com défice de vitamina $D$, sujeitos a uma intervenção diária com vitamina $\mathrm{D}$, não revelou efeitos nos parâmetros musculares. Baseado nestes resultados, aumentar as concentrações séricas de 25(OH)D de $20 \mathrm{ng} /$ $\mathrm{mL}$ para $>30 \mathrm{ng} / \mathrm{mL}$ não parece ter benefício na função muscular. É possível que doses mais elevadas ou um follow-up superior a 1 ano, pudessem modificar os resultados do estudo (20).

Tendo em conta o papel importante da proteína na função muscular em idosos com desnutrição e sarcopenia, incluíram-se 3 estudos com suplementos nutricionais enriquecidos com proteína e vitamina $D$. Verlaan S, et al. (21) observaram que, em idosos com sarcopenia, uma concentração de 25(OH)D acima de 50 nmol/L e uma ingestão proteica > 1,0 g/ $/ \mathrm{kg} /$ dia, contribui para ganhos superiores de MM. A vitamina $D$ pode agir de forma sinérgica com a leucina e a insulina na estimulação anabólica da síntese proteica.

Com a mesma intervenção e associada a um programa de atividade física, Englund A. D, et al. (22) concluíram que a adição da suplementação nutricional a longo-prazo resultou em maiores declínios na gordura intermuscular e melhoria da densidade muscular em idosos com mobilidade limitada e vitamina $D$ insuficiente $(9-24 \mathrm{ng} / \mathrm{mL}$ ). Este estudo revelou ainda que podem ser alcançadas melhorias na composição muscular, sem alteração substancial na perda de peso, muitas vezes verificada nestes doentes. De encontro a esses resultados, Yamada M, et al. (23) confirmaram o efeito sinérgico da suplementação de proteína com vitamina $\mathrm{D}$ e do exercício de resistência na qualidade muscular $\mathrm{e}$ FM, em idosos sarcopenicos.

Um estudo recente revelou que a prevalência de carência de vitamina $D$ em idosos malnutridos rondou quase os $60 \%$, sendo $20 \%$ considerada severa. Nos doentes com carência, a taxa de mortalidade em 180 dias aumentou de 23,1\% para 29,9\%. Alguns doentes receberam tratamento com vitamina $\mathrm{D}$, antes da admissão hospitalar ou durante o internamento, o que diminuiu o risco de mortalidade. Estes resultados sugerem que a elevada prevalência em doentes malnutridos, pode estar associada à severidade da doença, que compromete a exposição solar, e à ingestão alimentar inadequada com baixa quantidade de vitamina D (24).

Um ECR recente sobre o impacto da suplementação de vitamina $D$ na resposta à vacina contra influenza em idosos com carência de vitamina $D(<30 \mathrm{ng} / \mathrm{L})$, mostrou que esta promove um aumento do nível plasmático do TGF $\beta$, sem melhorar a produção de anticorpos. Para além disso, sugeriu que a suplementação parece direcionar a polarização dos linfócitos para uma tolerância à resposta imune (25). Da mesma forma, num outro ECR, uma suplementação mensal de uma dose elevada de vitamina $D$ reduziu em $40 \%$ a incidência de IRA em idosos residentes em cuidados prolongados, em comparação com um grupo de uma dose standard. A dose elevada não manifestou efeitos negativos na hipercalcemia, cálculos renais, hipervitaminose D, internamento, morte ou fraturas, mas revelou uma maior incidência de quedas. É evidente que o papel da suplementação de vitamina D na imunidade antiviral contra infeções respiratórias provavelmente depende do nível de vitamina D do indivíduo (26).

Sluyter D. J, et al.(27) e Martineau R. A, et al. (28) em dois ECR, também estudaram os efeitos de doses elevadas de vitamina $\mathrm{D}$ a longo-prazo na função pulmonar e na prevenção de IRA, respetivamente. No primeiro estudo, concentrações de $25(\mathrm{OH}) \mathrm{D}>50 \mathrm{nmol} / \mathrm{L}$, não melhoraram a função pulmonar, mas demonstraram melhorias em fumadores, principalmente com défice ou doenças pulmonares. No segundo estudo, também não se verificou influência na adição de doses intermitentes por bólus à suplementação standard nas IRA em idosos residentes em lares. Apesar disso, aumentou o risco e a duração das infeções do trato respiratório superior, concluindo que a suplementação diária é mais eficaz que a suplementação intermitente.

Um estudo de 2018 de Shimizu Y, et al. (29) destacou que a suplementação diária de vitamina $D$ reduz a duração e severidade da infeção respiratória e melhora a qualidade de vida dos doentes. A ingestão de $25(\mathrm{OH}) \mathrm{D}$ pode induzir proteção antimicrobiana e inibir a proliferação da infeção. O tamanho reduzido da amostra, a não examinação do comportamento clínico durante a ingestão e a subjetividade da sintomatologia reportada pelos doentes foram algumas das limitações do estudo.

No que diz respeito à COVID-19, llie C. P, et al. (30) relataram que os níveis de vitamina $D$ estão fortemente relacionados com o número de casos e especialmente com a taxa de mortalidade da COVID-19. Os idosos, população mais vulnerável, revelaram um maior défice. A evidente associação observada no presente estudo pode ser explicada pelo papel da vitamina D na prevenção da infeção por COVID-19 ou, mais provavelmente, por uma proteção potencial da vitamina $D$ nas consequências mais negativas da infeção.

Segundo um estudo de coorte, os doentes que apresentaram resultados positivos no teste do SARS-CoV-2, revelaram níveis mais baixos de vitamina $D$, sugerindo que o risco de infeção está fortemente relacionado com as concentrações de $25(\mathrm{OH}) \mathrm{D}$, mais do que em outras infeções respiratórias. Algumas limitações do estudo foram o número reduzido de doentes recolhidos de um único hospital, a indisponibilidade de informações clínicas sobre a severidade da sintomatologia e a possível influência da ingestão alimentar e da suplementação nos níveis de vitamina $D$ registados (31).

Asyary A, et al. (32) descobriram que uma maior duração da exposição à luz solar, tanto em meio hospitalar como domiciliar, estava relacionada a um maior número de casos recuperados de COVID-19 entre os doentes. Sabe-se que a luz solar desencadeia a produção de vitamina D, que funciona para aumentar a imunidade. Por outro lado, a luz solar pode manter a condição de saúde dos indivíduos, para que eles possam ter oportunidade de recuperar-se da doença.

Pelo contrário, Hastie C. et al. (33) numa coorte realizada em adultos e idosos do UK Biobank (37-73 anos), não forneceu evidências que as concentrações de vitamina $\mathrm{D}(25(\mathrm{OH}) \mathrm{D})$ possam estar associadas ao risco de infeção de COVID-19. No entanto, as concentrações de vitamina $D$ e o estado de saúde dos participantes foram avaliados no início do estudo, em vez de no momento do diagnóstico de COVID-19.

\section{CONCLUSÕES}

A presente revisão evidencia que os estudos têm resultados promissores na utilização da vitamina $D$ como suplemento nutricional, em caso de carência, na melhoria da função muscular, bem como redução da severidade e mortalidade por infeções respiratórias, acelerando o processo de recuperação e consequentemente diminuindo o tempo de internamento hospitalar. A combinação da vitamina D com a proteína e o exercício físico, potenciam os resultados a nível da preservação muscular e revelam um efeito sinérgico. Alguns estudos sugerem que doses elevadas de vitamina $D$ durante um curto período são eficazes no aumento das concentrações séricas de 25(OH)D, sem efeitos adversos em doentes com problemas respiratórios.

Por outro lado, alguns estudos não demonstraram uma relação benéfica evidente da suplementação de vitamina $D$ e relataram que a concentração pode ser influenciada entre outros fatores, pela localização geográfica, cor da pele, ingestão alimentar, exposição solar, estado nutricional e severidade das doenças. A elevada prevalência de carência de vitamina $\mathrm{D}$ em doentes com desnutrição, revelou estar associada à severidade da doença, que compromete a exposição solar, e à ingestão alimentar 
inadequada com baixa quantidade de vitamina $\mathrm{D}$. Em doentes desnutridos a intervenção nutricional deverá ser abrangente e não focada apenas num único nutriente, uma vez que é sabido que a desnutrição afeta vários sistemas e órgãos, sendo pouco provável que a suplementação de vitamina D por si só, tenha resultados relevantes na imunidade.

O aumento das taxas de mortalidade em doentes idosos malnutridos, nas quais foram relatadas concentrações mais baixas de 25(OH)D, podem suportar um possível papel benéfico da vitamina $D$ na recuperação e no risco de COVID-19. No entanto, a evidência existente é pouco conclusiva e não é suficiente para estabelecer uma relação direta entre a carência de vitamina $D$ e o risco de incorrer em COVID-19 no futuro. As maiores limitações relatadas nos estudos foram as amostras pequenas, a possível influência da dieta e da exposição solar nos resultados, o tempo reduzido de follow-up e a ausência de grupos de controlo, que analisassem outros fatores.

Em suma, a evidência disponível não permite concluir que a suplementação de vitamina D seja eficaz em idosos com COVID-19. A hipótese de que a suplementação de vitamina $\mathrm{D}$ possa reduzir o risco e a incidência de morte por COVID-19 na população idosa está ainda em investigação, pelo que devem ser efetuados ensaios clínicos para comprovar a veracidade e para determinar a eficácia e segurança em doentes com desnutrição e sarcopenia. A carência de vitamina D nos idosos, com base nos estudos efetuados na população portuguesa e no IAN-AF, é uma realidade que deve ser alvo do desenvolvimento de políticas de saúde pública para a sua correção.

\section{REFERÊNCIAS BIBLIOGRÁFICAS}

1. WHO. Coronavirus disease (COVID-19) Pandemic.

2. Guo YR, Cao QD, Hong ZS, Tan YY, Chen SD, Jin HJ, et al. The origin, transmission and clinical therapies on coronavirus disease 2019 (COVID-19) outbreak- A n update on the status. Vol. 7, Military Medical Research. BioMed Central Ltd.; 2020.

3. Niu S, Tian S, Lou J, Kang X, Zhang L, Lian H, et al. Clinical characteristics of older patients infected with COVID-19: A descriptive study. Arch Gerontol Geriatr. 2020 Jul;89:104058. 4. Cederholm T, Jensen GL, Correia MITD, Gonzalez MC, Fukushima R, Higashiguchi T, et al. GLIM criteria for the diagnosis of malnutrition - A consensus report from the global clinical nutrition community. Clin Nutr. 2019 Feb;38(1):1-9.

5. Cruz-Jentoft AJ, Bahat G, Bauer J, Boirie Y, Bruyère O, Cederholm T, et al. Sarcopenia: Revised European consensus on definition and diagnosis. Vol. 48, Age and Ageing. Oxford University Press; 2019. p. 16-31.

6. Sieber CC. Malnutrition and sarcopenia. Aging Clin Exp Res. 2019;31(6):793-8. 7. Calder PC, Carr AC, Gombart AF, Eggersdorfer M. Optimal nutritional status for a well-functioning immune system is an important factor to protect against viral infections. Vol. 12, Nutrients. MDPI AG; 2020.

8. Derbyshire E, Delange J. COVID-19: is there a role for immunonutrition, particularly in the over 65s? BMJ Nutr Prev Heal. 2020 Apr;bmjnph-2020-000071.

9. Deutz NEP, Bauer JM, Barazzoni R, Biolo G, Boirie Y, Bosy-Westphal A, et al. Protein intake and exercise for optimal muscle function with aging: Recommendations from the ESPEN Expert Group. Clin Nutr. 2014 Dec;33(6):929-36.

10. Gombart AF, Pierre A, Maggini S. A review of micronutrients and the immune system-working in harmony to reduce the risk of infection. Nutrients. 2020 Jan;12(1). 11. Jayawardena R, Sooriyaarachchi $P$, Chourdakis M, Jeewandara $C$, Ranasinghe $P$. Enhancing immunity in viral infections, with special emphasis on COVID-19: A review. Diabetes Metab Syndr Clin Res Rev. 2020 Jul;14(4):367-82.

12. Martineau AR, Jolliffe DA, Greenberg L, Aloia JF, Bergman P, Dubnov-Raz G, et al. Vitamin $D$ supplementation to prevent acute respiratory infections: Individual participant data meta-analysis. Health Technol Assess (Rockv). 2019 Jan;23(2):1-44.

13. Grant WB, Lahore H, McDonnell SL, Baggerly CA, French CB, Aliano JL, et al. Evidence that vitamin d supplementation could reduce risk of influenza and covid-19 infections and deaths. Vol. 12, Nutrients. MDPI AG; 2020.

14. Duarte C, Carvalheiro H, Rodrigues AM, Dias SS, Marques A, Santiago T, et al.
Prevalence of vitamin D deficiency and its predictors in the Portuguese population: a nationwide population-based study. Arch Osteoporos. 2020 Mar 2;15(1):36. doi: 10.1007/s11657-020-0695-x.

15. Santos A, Amaral TF, Guerra RS, Sousa AS, Álvares L, Moreira P, et al. Vitamin $D$ status and associated factors among Portuguese older adults: Results from the Nutrition UP 65 cross-sectional study. BMJ Open. 2017 Jun 1;7(6):e016123.

16. Carla Lopes, Duarte Torres, Andreia Oliveira, Milton Severo, Violeta Alarcão, Sofia Guiomar, et al. Inquérito Alimentar Nacional e de Atividade Física, IAN-AF 2015-2016. 2017. Available from: https://ian-af.up.pt/sites/default/files/IAN-AF Relatório Resultados_0.pdf

17. National Academy of Sciences. Dietary Reference Intakes (DRIs): Recommended Dietary Allowances and Adequate Intakes, Vitamins. 2011.

18. El Hajj C, Fares S, Chardigny JM, Boirie Y, Walrand S. Vitamin D supplementation and muscle strength in pre-sarcopenic elderly Lebanese people: a randomized controlled trial. Arch Osteoporos. 2019 Dec;14(1).

19. Aoki K, Sakuma M, Endo N. The impact of exercise and vitamin D supplementation on physical function in community-dwelling elderly individuals: A randomized trial. J Orthop Sci. 2018 Jul;23(4):682-7.

20. Shea MK, Fielding RA, Dawson-Hughes B. The effect of vitamin D supplementation on lower-extremity power and function in older adults: a randomized controlled trial. PubMed - NCBI. The American Journal of Clinical Nutrition. 2019. p. 369-79.

21. Verlaan S, Maier AB, Bauer JM, Bautmans I, Brandt K, Donini LM, et al. Sufficient levels of 25-hydroxyvitamin $D$ and protein intake required to increase muscle mass in sarcopenic older adults - The PROVIDE study. Clin Nutr. 2018 Apr;37(2):551-7.

22. Englund DA, Kirn DR, Koochek A, Zhu H, Travison TG, Reid KF, et al. Nutritional Supplementation With Physical Activity Improves Muscle Composition in MobilityLimited Older Adults, The VIVE2 Study: A Randomized, Double-Blind, PlaceboControlled Trial. J Gerontol A Biol Sci Med Sci. 2017 Dec;73(1):95-101.

23. Yamada M, Kimura Y, Ishiyama D, Nishio N, Otobe Y, Tanaka T, et al. Synergistic effect of bodyweight resistance exercise and protein supplementation on skeletal muscle in sarcopenic or dynapenic older adults. Geriatr Gerontol Int. 2019 May; 19(5):429-37. 24. Merker M, Amsler A, Pereira R, Bolliger R, Tribolet P, Braun N, et al. Vitamin D deficiency is highly prevalent in malnourished inpatients and associated with higher mortality: A prospective cohort study. Med (United States). 2019 Nov;98(48).

25. Goncalves-Mendes N, Talvas J, Dualé C, Guttmann A, Corbin V, Marceau G, et al. Impact of Vitamin $D$ supplementation on influenza vaccine response and immune functions in deficient elderly persons: A randomized placebo-controlled trial. Front Immunol. 2019;10(FEB).

26. Ginde AA, Blatchford P, Breese K, Zarrabi L, Linnebur SA, Wallace Jl, et al. High-Dose Monthly Vitamin D for Prevention of Acute Respiratory Infection in Older Long-Term Care Residents: A Randomized Clinical Trial. J Am Geriatr Soc. 2017 Mar;65(3):496-503.

27. Sluyter JD, Camargo CA, Waayer D, Lawes CMM, Toop L, Khaw KT, et al. Effect of monthly, high-dose, long-term vitamin $\mathrm{D}$ on lung function: A randomized controlled trial. Nutrients. 2017 Dec;9(12).

28. Martineau AR, Hanifa Y, Witt KD, Barnes NC, Hooper RL, Patel M, et al. Double-blind randomised controlled trial of vitamin $\mathrm{D} 3$ supplementation for the prevention of acute respiratory infection in older adults and their carers (ViDiFlu). Thorax. 2015 Oct;70(10):953-60. 29. Shimizu Y, Ito Y, Yui K, Egawa K, Orimo H. Intake of 25-hydroxyvitamin D3 reduces duration and severity of upper respiratory tract infection: A randomized, doubleblind, placebo-controlled, parallel group comparison study. J Nutr Heal Aging. 2018 Apr;22(4):491-500.

30. llie PC, Stefanescu S, Smith L. The role of vitamin D in the prevention of coronavirus disease 2019 infection and mortality. Aging Clin Exp Res. 2020 May;1-4.

31. D'Avolio A, Avataneo V, Manca A, Cusato J, De Nicolò A, Lucchini R, et al. 25-Hydroxyvitamin D Concentrations Are Lower in Patients with Positive PCR for SARS-CoV-2. Nutrients. 2020 May;12(5):1359.

32. Asyary A, Veruswati M. Sunlight exposure increased Covid-19 recovery rates: A study in the central pandemic area of Indonesia. Sci Total Environ. 2020 Aug;729:139016. 33. Hastie CE, Mackay DF, Ho F, Celis-Morales CA, Katikireddi SV, Niedzwiedz CL, et al. Vitamin D concentrations and COVID-19 infection in UK Biobank. Diabetes Metab Syndr Clin Res Rev. 2020 Jul;14(4):561-5. 